\title{
Nucleon Polarizabilities
}

\author{
Barry R. Holstein \\ Department of Physics-LGRT \\ University of Massachusetts \\ Amherst, MA 01003
}

October 23, 2018

\begin{abstract}
The subject of nucleon polarizabilities in real, virtual, and doubly virtual Compton scattering is discussed with respect to what is known and how such quantitities can be extracted from data.
\end{abstract}




\section{Introduction}

The subject of nucleon polarizabilities - both real and virtual-has become a hot one lately with activity on both the experimental and theoretical fronts. In this article, we present a brief review of some of the interesting issues, including the latest experimental results as well as new developments on the theoretical front, wherein dispersion relations allow extraction of "higher order" nucleon polarizabilities as well as connections to be made between the GDH sum rule for real photons and the Bjorken sum rule for high $Q^{2}$.

\section{RCS: Ordinary Polarizabilities}

Historically, the basic idea of polarizabilities and their meaning comes from the idea of the response of a system to the application of an external quasistatic electromagnetic field[1]. For example, in the presence of an external electric field $\vec{E}_{0}$ a system of charges will in general deform, with positive charges moving one way and negative charges the other, resulting in an induced dipole moment $\vec{p}$ whose size is proportional to the strength of the applied field

$$
\vec{p}=4 \pi \alpha_{E} \vec{E}_{0}
$$

The electric polarizability $\alpha_{E}$ is then the constant of proportionality between the applied field and the induced dipole moment. Similarly one can define the magnetic polarizability $\beta_{M}$ in terms of the induced magnetic dipole moment $\vec{\mu}$ in the presence of an external mmagnetizing field $\vec{H}_{0}$

$$
\vec{\mu}=4 \pi \beta_{M} \vec{H}_{0}
$$

For a macroscopic system it is intuitively clear how to measure such properties, but for an elementary particle, one must generate the electric and magnetic fields via photons and use Compton scattering in order to provide quantification. In order to see how this is done first consider RCS at very low energy - say $\omega<<20 \mathrm{MeV}$ - wherein the photon wavelength is much longer than the size of the nucleon. In this case, one is unable to resolve the structure of the target and is sensitive only to its overall charge $-e-$ and mass $-m$. The interaction is then described by the simple lowest order Hamiltonian

$$
H=\frac{(\vec{p}-e \vec{A})^{2}}{2 m}+e \phi
$$


and the resultant Compton scattering amplitude has the canonical Thomson form

$$
\operatorname{Amp}=-\frac{e^{2}}{m} \hat{\epsilon}^{\prime} \cdot \hat{\epsilon}
$$

At higher energy - shorter wavelength - the internal structure becomes visible and one can describe the interaction in terms of an effective Hamiltonian having certain elementary properties -

i) quadratic in $\vec{A}$;

ii) gauge invariant;

iii) rotational scalar;

iv) $\mathrm{P}, \mathrm{T}$ even, etc.

To the next leading order then the resultant form of the interaction is unique and must have the from

$$
H_{e f f}=-\frac{1}{2} 4 \pi \alpha_{E} \vec{E}^{2}-\frac{1}{2} 4 \pi \beta_{M} \vec{H}^{2}
$$

where $\alpha_{E}, \beta_{M}$ are just the electric, magnetic polarizabilities defined above, as can be seen from the definitions

$$
\vec{p}=-\frac{\delta H_{e f f}}{\delta \vec{E}}=4 \pi \alpha_{E} \vec{E} ; \quad \vec{\mu}=-\frac{\delta H_{e f f}}{\delta \vec{H}}=4 \pi \beta_{M} \vec{H}
$$

Using the effective Hamiltonians given above the Compton scattering amplitude becomes

$$
\operatorname{Amp}^{(2)}=\hat{\epsilon} \cdot \hat{\epsilon}^{\prime}\left(\frac{-e^{2}}{M}+\omega \omega^{\prime} 4 \pi \alpha_{E}^{p}\right)+\hat{\epsilon} \times \vec{k} \cdot \hat{\epsilon}^{\prime} \times \vec{k}^{\prime} 4 \pi \beta_{M}^{p}+\mathcal{O}\left(\omega^{4}\right) .
$$

and the resultant differential scattering cross section is

$$
\begin{aligned}
\frac{d \sigma}{d \Omega} & =\left(\frac{\alpha}{M}\right)^{2}\left(\frac{\omega^{\prime}}{\omega}\right)^{2}\left[\frac{1}{2}\left(1+\cos ^{2} \theta\right)\right. \\
& \left.-\frac{M \omega \omega^{\prime}}{\alpha}\left(\frac{1}{2}\left(\alpha_{E}^{p}+\beta_{M}^{p}\right)(1+\cos \theta)^{2}+\frac{1}{2}\left(\alpha_{E}^{p}-\beta_{M}^{p}\right)(1-\cos \theta)^{2}\right)+\ldots\right]
\end{aligned}
$$


here $\alpha=e^{2} / 4 \pi$ is the fine structure constant. It is clear then that $\alpha_{E}, \beta_{M}$ can be extracted via careful measurement of the differential cross section and previous experiements at SAL and MAMI have yielded the values [2]

$$
\alpha_{E}^{p}=(12.1 \pm 0.8 \pm 0.5) \times 10^{-4} \mathrm{fm}^{3} ; \quad \beta_{M}^{p}=(2.1 \mp 0.8 \mp 0.5) \times 10^{-4} \mathrm{fm}^{3} .
$$

Recently preliminary new values have been announced obtained from precise $p(\gamma, \gamma) p$ measurements using the TAPS and LARA spectrometers [3]

$\alpha_{E}^{p}=(12.24 \pm 0.24 \pm 0.54) \times 10^{-4} \mathrm{fm}^{3} ; \quad \beta_{M}^{p}=(1.57 \mp 0.24 \mp 0.54) \times 10^{-4} \mathrm{fm}^{3}$.

Hemmert will show how these numbers compare with values obtained via chiral perturbation theory.

The above results are well known and our task today is to extend this discussion to indlude spin degrees of freedom. In this case the general Compton amplitude can written in the general form

$$
\begin{aligned}
T & =A_{1}(\omega, z) \vec{\epsilon}^{\prime} \cdot \vec{\epsilon}+A_{2}(\omega, z) \vec{\epsilon}^{\prime} \cdot \hat{k} \vec{\epsilon} \cdot \hat{k}^{\prime} \\
& +i A_{3}(\omega, z) \vec{\sigma} \cdot\left(\vec{\epsilon}^{\prime} \times \vec{\epsilon}\right)+i A_{4}(\omega, z) \vec{\sigma} \cdot\left(\hat{k}^{\prime} \times \hat{k}\right) \vec{\epsilon}^{\prime} \cdot \vec{\epsilon} \\
& +i A_{5}(\omega, z) \vec{\sigma} \cdot\left[\left(\vec{\epsilon}^{\prime} \times \hat{k}\right) \vec{\epsilon} \cdot \hat{k}^{\prime}-\left(\vec{\epsilon} \times \hat{k}^{\prime}\right) \vec{\epsilon}^{\prime} \cdot \hat{k}\right] \\
& +i A_{6}(\omega, z) \vec{\sigma} \cdot\left[\left(\vec{\epsilon}^{\prime} \times \hat{k}^{\prime}\right) \hat{\epsilon} \cdot \hat{k}^{\prime}-(\vec{\epsilon} \times \hat{k}) \vec{\epsilon}^{\prime} \cdot \hat{k}\right],
\end{aligned}
$$

and each amplitude can be expanded in terms of a lowest order Born contribution plus a higher order and structure dependent polarizability term. In the case of the spin-dependent amplitudes $A_{3,4,5,6}$ such structure effects arise at $\mathcal{O}\left(\omega^{3}\right)$ and can be characterized in terms of an effective Hamiltonian involving four "spin-polarizabilities"

$$
H_{e f f}^{(3)}=-\frac{1}{2} 4 \pi\left(\gamma_{E 1}^{p} \vec{\sigma} \cdot \vec{E} \times \dot{\vec{E}}+\gamma_{M 1}^{p} \vec{\sigma} \cdot \vec{H} \times \dot{\vec{H}}-2 \gamma_{E 2}^{p} E_{i j} \sigma_{i} H_{j}+2 \gamma_{M 2}^{p} H_{i j} \sigma_{i} E_{j}\right)
$$

where

$$
E_{i j}=\frac{1}{2}\left(\nabla_{i} E_{j}+\nabla_{j} E_{i}\right), \quad H_{i j}=\frac{1}{2}\left(\nabla_{i} H_{j}+\nabla_{j} H_{i}\right)
$$

denote electric and magnetizing field gradients. While these quantities are mathematically well-defined via Eq. 12, I am unable to provide a good physical picture. The parameters $\gamma_{E 1}, \gamma_{M 1}$ are related to the classical Faraday

\footnotetext{
${ }^{1}$ In order to put these numbers in perspective note that for a hydrogen atom one finds $\alpha_{E}(H) \sim \operatorname{Volume}(H)$ while for the proton Eq. 9 gives $\alpha_{E}(p) \sim 10^{-3} \operatorname{Volume}(p)$, so that the proton is a much more strongly bound system.
} 
rotation, wherein the linear polarization of the photon passing longitudinally through a magnetized medium exhibits a rotation due to the difference in index of refraction for photons with circular polarization parallel and antiparallel to the direction of magnetization. However, I don't know how to go much farther than this and will offer a bottle of fine German wine to anyone who is able to provide me such a classical picture.

Again I will rely on Thomas Hemmert to present the theoretical situation, but on the experimental side, there exist as yet no direct polarized Compton scattering measurements. However, a global analysis of unpolarized Compton data by the LEGS group has yielded the value 4$]^{2}$

$$
\gamma_{\pi}=-\gamma_{E 1}-\gamma_{M 2}+\gamma_{E 2}+\gamma_{M 1}=(15.7 \pm 2.3 \pm 2.8 \pm 2.4) \times 10^{-4} \mathrm{fm}^{4}
$$

in disagreement with the theoretical prediction

$$
\gamma_{\pi}^{t h}=\frac{\alpha g_{A}^{2}}{96 \pi^{2} F_{\pi}^{2} m_{\pi}^{2}}\left(4-\left(\frac{9}{2}+\kappa_{n}\right) \frac{\pi m_{\pi}}{M}\right)=3.3 \times 10^{-4} \mathrm{fm}^{4}
$$

from $\mathcal{O}\left(p^{4}\right)$ heavy baryon chiral perturbation theory [5]. However, a preliminary new value has been announced from the TAPS data

$$
\gamma_{\pi}=(7.4 \pm 2.3) \times 10^{-4} \mathrm{fm}^{4}
$$

which is in better agreement with theory. The other quantity about which much has been written is the forward spin polarizability $\gamma_{0}$, which is given by the first moment of the DGH sum rule

$$
\gamma_{0}=-\gamma_{E 1}-\gamma_{M 2}-\gamma_{E 2}-\gamma_{M 1}=\int_{\omega_{0}}^{\infty} \frac{d \omega}{\omega^{3}}\left(\sigma_{\frac{3}{2}}(\omega)-\sigma_{\frac{1}{2}}(\omega)\right)
$$

Drechsel has quoted perhaps the best current value of the sum rule, based upon the MAID analysis,

$$
\gamma_{0}=-0.75 \times 10^{-4} \mathrm{fm}^{4}
$$

which is in reasonable agreement with previous determinations.

While at present we do not have direct experimental values for the four spin-polarizabilities, it may be possible to extract them from future $\vec{p}(\vec{\gamma}, \gamma) p$

\footnotetext{
${ }^{2}$ Note here that we have subtracted the pion pole contribution.
} 


$\begin{array}{ccc}\text { polarizability } & \text { HB } \chi \mathrm{pt} & \text { Dispersive Evaluation } \\ \gamma_{E 1}^{p} & -1.8 & -4.4 \\ \gamma_{M 1}^{p} & 2.9 & 2.9 \\ \gamma_{E 2}^{p} & 1.8 & 2.2 \\ \gamma_{M 2}^{p} & 0.7 & 0.0\end{array}$

Table 1: Calculated and "experimental" values for spin polarizabilities obtained via dispersion relations. All are in units of $10^{-4} \mathrm{fm}^{4}$.

studies. In the meantime, it has been realized that they can be obtained using a dispersive analysis of the Compton process [6]. One assumes that the Compton amplitudes $A_{i}$ can be represented in terms of once subtracted dispersion relations at fixed $\mathrm{t}$

$$
A_{i}(\nu, t)=A_{i}^{\text {Born }}(\nu, t)+\left(A_{i}(0, t)-A_{i}^{\text {Born }}(0, t)\right)+\frac{2 \nu^{2}}{\pi} P \int_{\nu_{t h r}}^{\infty} \frac{\operatorname{Im} A_{i}\left(\nu^{\prime}, t\right)}{\nu^{\prime}\left(\nu^{\prime 2}-\nu^{2}\right)} .
$$

Here $\operatorname{Im} A_{i}\left(\nu^{\prime}, t\right)$ is evaluated using empirical photoproduction data while the subtraction constant $A_{i}(0, t)-A_{i}^{\mathrm{Born}}(0, t)$ is represented via use of $\mathrm{t}$-channel dispersion relations

$$
A_{i}(0, t)-A_{i}^{\text {Born }}(0, t)=a_{i}+a_{i}^{t-\text { pole }}+\frac{t}{\pi}\left(\int_{4 m_{\pi}^{2}}^{\infty}-\int_{-\infty}^{-4 M m_{\pi}-2 m_{\pi}^{2}} d t^{\prime} \frac{\operatorname{Im}_{t} A_{i}\left(0, t^{\prime}\right)}{t^{\prime}\left(t^{\prime}-t\right)}\right)
$$

with $\operatorname{Im}_{t} A_{i}$ evaluated using the contribution from the $\pi \pi$ intermediate state. In principle then there remain six unknown subtraction constants $a_{i}$ to be determined empirically. However, in view of the limitations posed by the the data, Drechsel et al. note that four of these quantities can be reasonably assumed to obey unsubtracted forward dispersion relations, while the remaining two $-\alpha_{E}-\beta_{M}$ and $\gamma_{\pi}$ - can be determined by an experimental fit. Once this is done the other spin polarizabilities may be extracted using sum rules, as done above in the case of the forward spin polarizability. The results of this process are given in Table 1, where they are compared to the numbers calculated in the $\mathcal{O}\left(p^{4}\right)$ heavy baryon chiral perturbation theory calculation described by Hemmert.

It has been noted by Babusci et al.[7] and by Holstein et al. [8] that one can extend this analysis to include terms of $\mathcal{O}\left(\omega^{4}\right)$ in the Compton amplitude 
by introducing higher order polarizabilities via

$$
H_{e f f}^{(4)}=-\frac{1}{2} 4 \pi \alpha_{E \nu}^{p} \dot{\vec{E}}^{2}-\frac{1}{2} 4 \pi \beta_{M \nu}^{p} \dot{\vec{H}}^{2}-\frac{1}{12} 4 \pi \alpha_{E 2}^{p} E_{i j}^{2}-\frac{1}{12} 4 \pi \beta_{M 2}^{p} H_{i j}^{2}
$$

Likewise Holstein et al. have extended this to $\mathcal{O}\left(\omega^{5}\right)$ by defining higher order spin-polarizabilities-

$$
\begin{aligned}
H_{e f f}^{(5)} & =-\frac{1}{2} 4 \pi\left[\gamma_{E 1 \nu}^{p} \vec{\sigma} \cdot \dot{\vec{E}} \times \ddot{\vec{E}}+\gamma_{M 1 \nu}^{p} \vec{\sigma} \cdot \dot{\vec{H}} \times \ddot{\vec{H}}-2 \gamma_{E 2 \nu}^{p} \sigma_{i} \dot{E}_{i j} \dot{H}_{j}+2 \gamma_{M 2 \nu}^{p} \sigma_{i} \dot{H}_{i j} \dot{E}_{j}\right. \\
& \left.+4 \gamma_{E T}^{p} \epsilon_{i j k} \sigma_{i} E_{j \ell} \dot{E}_{k \ell}+4 \gamma_{M T}^{p} \epsilon_{i j k} \sigma_{i} H_{j \ell} \dot{H}_{k \ell}-6 \gamma_{E 3}^{p} \sigma_{i} E_{i j k} H_{j k}+6 \gamma_{M 3}^{p} \sigma_{i} H_{i j k} E_{j k}\right] \$
\end{aligned}
$$

where

$$
\begin{aligned}
(E, H)_{i j k} & =\frac{1}{3}\left(\nabla_{i} \nabla_{j}(E, H)_{k}+\nabla_{i} \nabla_{k}(E, H)_{j}+\nabla_{j} \nabla_{k}(E, H)_{i}\right) \\
& -\frac{1}{15}\left(\delta_{i j} \nabla^{2}(E, H)_{k}+\delta_{j k} \nabla^{2}(E, H)_{i}+\delta_{i k} \nabla^{2}(E, H)_{j}\right)
\end{aligned}
$$

are the (spherical) tensor gradients of the electric and magnetizing fields. Each of these new higher order polarizabilities can be extracted via sum rules from the Mainz dispersive analysis and results are given Table 2

Again Hemmert will discuss the details it is clear that the chiral perturbation theory description is remarkably successful in describing all of these properties - the pion cloud plays the dominant role in determining the polarizabilities.

\section{VCS: Generalized Polarizabilities}

There has recently developed an interest in the process of virtual Compton scattering process by which one can measure "generalized" (q-dependent) polarizabilities 9]. In order to understand the meaning of such quantities, recall that in ordinary electron scattering measurement of the q-dependent charge form factor allows access, via Fourier transform, to the nucleon charge density. In an analogous fashion measurement of a generalized polarizability such as $\alpha_{E}(q)$ permits one to determine the polarization density of the nucleon. On the experimental side this is an extremently challenging process because the generalized polarizabilities can be determined only after (large) 


$\begin{array}{ccc}\text { polarizability } & \text { HB } \chi \mathrm{pt} & \text { Dispersive value } \\ \alpha_{E \nu}^{p} & 2.4 & -3.8 \\ \beta_{M \nu}^{p} & 7.5 & 9.3 \\ \alpha_{E 2}^{p} & 22.1 & 29.3 \\ \beta_{M 2}^{p} & -9.5 & -24.3 \\ \gamma_{E 1 \nu}^{p} & -2.4 & -3.4 \\ \gamma_{M 1 \nu}^{p} & 1.8 & 2.2 \\ \gamma_{E 2 \nu}^{p} & 1.6 & 1.3 \\ \gamma_{M 2 \nu}^{p} & -0.1 & -0.6\end{array}$

Table 2: Calculated and "experimental" values for higher order polarizabilities obtained via dispersion relations. Spin independent and spin dependent polarizabilities are in units of $10^{-4} \mathrm{fm}^{5}$ and $10^{-4} \mathrm{fm}^{6}$ respectively

Bethe-Heitler and Born diagram contributions have been subtracted. One then seeks a systematic deviation - growing with $\omega^{\prime}$-of the measured cross section from that predicted with only Bethe-Heitler plus Born input in order to extract the desired signal. This has been achieved in a recent MAMI experiment 10] and what results is information on the two combinations

$$
\begin{aligned}
P_{L L}-\frac{1}{\epsilon} P_{T T} & =a_{0} \alpha_{E}(q)-c_{1} \gamma_{M 2}(q)+c_{2} M^{M 1-M 1}(q) \\
P_{L T} & =b_{0} \beta_{M}(q)+c_{3} M^{C 0-M 1}(q)-c_{4} \gamma_{E 2}(q)
\end{aligned}
$$

where here the multipoles $M^{M 1-M 1}, M^{C 0-M 1}$ have no RCS analogs. The extracted numbers are given in Table 3 together with values calculated in various models as well as in $\mathrm{HB} \chi \mathrm{pt}$. It is remarkable that once again agreement with the simple chiral calculation is outstanding, despite the fact that the measurement took place at $q=0.6 \mathrm{GeV}$, where one questions the validity of the chiral approach.

Fortunately, there is an additional experiment underway which should help in this regard 15]. A Bates measurement using the OOPS spectrometer system has just begun taking data and will yield values of the generalized polarizabilities at $q=230 \mathrm{MeV}$, where the chiral predictions should work.

\footnotetext{
${ }^{3}$ Radiative corrections are also substantial here.
} 


\begin{tabular}{c|c|c} 
& $P_{L L}-P_{T T} / \epsilon$ & $P_{L T}$ \\
\hline & \multicolumn{2}{|c}{} \\
expt. & $23.7 \pm 2.2 \pm 0.6 \pm 4.3$ & $-5.0 \pm 0.8 \pm 1.1 \pm 1.4$ \\
HB $\chi \mathrm{pt} 11]$ & 26.0 & -5.3 \\
L $\sigma \mathrm{M}[12$ & 11.5 & 0.0 \\
ELM 13 & 5.9 & -1.9 \\
NRQM 13 & 11.1 & -3.5
\end{tabular}

Table 3: Measured and calculated values for generalized polarizabilities. All are in units of $\mathrm{GeV}^{-2}$.

Another interesting feature of this measurement is that is it employs perpendicular kinematics (the lepton and hadron planes are orthogonal) for which the Bethe-Heitler plus Born "background" should be much less significant, allowing more straightforward access to the desired generalized polarizabilities.

On the theoretical side, an extension of the Mainz dispersive RCS analysis to the case of VCS has been done 16. This calculation is not as straightforward as it might appear, since replacement of a real photon by a virtual one requires now twelve invariant amplitudes which are functions of three variables, which may be taken as $\omega, \theta, Q^{2}$. Analysis of the asymptotic dependence is correspondingly much more complex. In addition the subtraction "constants" are now functions of $Q^{2}$ whose form must be assumed. The calculation is still in progress but preliminary results for four of the generalized polarizabilities compard to the chiral predictions are available. Agreement is satisfactory though not outstanding, but Hemmert has argued that $\mathcal{O}\left(p^{4}\right)$ corrections will improve matters.

\section{VVCS: the GDH Sum Rule}

My final topic will be one that is not usually described in terms of polarizabilities, but could be - that of doubly virtual Compton scattering. This process is also virtual, in that it is not really experimentally feasible. Nevertheless, it is of great theoretical interest, as we shall see. In order to make things 
a simple as possible we consider forward scattering with virtual photons of identical $q^{2}$, for which the anti-symmetric component of the scattering tensor can be written in the form[17]

$$
\begin{aligned}
T_{\mu \nu}^{a n t i} & =i \int d^{4} x e^{i q \cdot x}<N_{p, s} \mid T\left(J_{\mu}(x) J_{\nu}(0) \mid N_{p, s}>_{a n t i}\right. \\
& =-i \epsilon_{\mu \nu \alpha \beta} q^{\alpha}\left[S^{\beta} S_{1}\left(\nu, Q^{2}\right)+\left(\nu S_{\beta}-S-\cdot q \frac{P^{\beta}}{M}\right) S_{2}\left(\nu, Q^{2}\right)\right]
\end{aligned}
$$

where, as usual, $\nu=p \cdot q / M$ and $S^{\beta}$ is the Pauli-Lubinski spin vector. By Regge or QCD arguments the sum rule for the non-Born component of the form factor $S_{1}$ should converge, so we have

$$
\frac{1}{4} S_{1}\left(0, Q^{2}\right)=\int_{\nu_{0}}^{\infty} \frac{d \nu}{\nu} G_{1}\left(\nu, Q^{2}\right) \equiv \frac{1}{M^{2}} I\left(Q^{2}\right)=\frac{2 M^{2}}{Q^{2}} \Gamma\left(Q^{2}\right)
$$

What is interesting here is that in the real photon limit we have

$$
I^{p, n}\left(Q^{2}\right)=-\frac{1}{4} \kappa_{p, n}^{2}
$$

where $\kappa_{p, n}$ is the anomalous moment so that we recognize Eq. 25 as being the Gerasimov-Drell-Hearn sum rule[18]. On the other hand, in the limit as $Q^{2} \rightarrow \infty$ er jsbr

$$
\Gamma\left(Q^{2}\right)^{p, n} \rightarrow \int_{0}^{1} d x g_{1}\left(x, Q^{2}\right) \rightarrow \pm \frac{1}{12} g_{A}+\frac{1}{36} g_{8}+\frac{1}{9} g_{0}
$$

where $g_{A}, g_{8}, g_{0}$ are the usual octet axial couplings, so that we recognize

$$
\Gamma^{p}\left(Q^{2}\right)-\Gamma^{n}\left(Q^{2}\right) \stackrel{Q^{2} \rightarrow \infty}{\longrightarrow} \frac{1}{6} g_{A}
$$

as being the Bjorken sum rule[19]. Now, as argued by Ji and Osborne[17], QCD (operator product expansion) methods can be used to extend the Bjorken sum rule down to values of $Q^{2}$ as low as $\sim 0.5 \mathrm{GeV}^{2}$ while chiral perturbation theory methods can be used in order to go from the real photon point at $Q^{2}=0$ up to higher values $\sim \leq 0.1 \mathrm{GeV}^{2}$ so the challenge is to connect the two pictures and work on this is ongoing. In this context it is interesting to make two comments: 
i) Much has been made of the possible discrepancy between the theoretical and experimental values of the GDH sum rule for the difference of neutron and proton, while no one questions the convergence of the Bjorken sum rule. It seems to me that if one is true then so must be the other.

ii) One can make a connection here with the spin-polarizability by calculating the weighted integral

$$
\int_{\nu_{0}}^{\infty} \frac{d \nu}{\nu^{3}} G_{1}\left(\nu, Q^{2}\right)
$$

but as yet this has not been looked at.

There is much more to be said on both issues.

\section{Conclusion}

We have seen above that the subject of nucleon polarizabilities is now a hot topic of research. In the case of real Compton scattering, the ordinary electric and magnetic polarizabilities are now well known and the challenge is to extend such measurements to the higher order spin polarizabilities. In the mean time, dispersion relations combined with sum rules can provide values for these numbers. In the case of virtual Compton scattering, the soon to be finalized generalized polarizbilities from the Mainz experiment are already challenging theoretical models and we anxiously await results from ongoing JLab and Bates experiments. Finally, in the case of doubly virtual Compton scattering, connections can be made between sacred sum rules for both QCD and electromagnetic physics. This is indeed an exciting time for Compton scatterers.

\section{Acknowledgement}

It is a pleasure to acknowledge the hospitality of the Chiral Dynamics2000 organizers. This work was supported in part by the National Science Foundation. 


\section{References}

[1] B.R. Holstein, Comm. Nucl. Part. Phys. 19, 221 (1990).

[2] F.J. Federspiel et al., Phys. Rev. Lett. 67, 1511 (1991); E.L. Hallin et al., Phys. Rev. C48, 1497 (1993); A. Zieger et al., Phys. Lett. B278, 34 (1992); B.E. MacGibbon et al., Phys. Rev. C52, 2097 (1995).

[3] F. Wissmann, proc. GDH2000, to be published, Göttingen Habilitationsscrift, April 2000.

[4] J.I. Tonnison, A.M. Sandorfi, S. Hoblit, and A.M. Nathan, Phys. Rev. Lett. 80, 4382 (1998).

[5] G.C. Gellas, T.R. Hemmert, and U.-G. Meissner, Phys. Rev. Lett. 85, 14 (2000); see, also, K.B.V. Kumar, J.A. McGovern, and M.C. Birse, Phys. Lett. B479, 167 (2000).

[6] D. Drechsel, M. Gorchtein, B. Pasquini, and M. Vanderhaeghen, Phys. Rev. C61, 015204 (2000).

[7] D. Babusci, G. Giordano, A.I. L'vov, G. Matone, and A.M. Nathan, Phys. Rev. C58, 1013 (1998).

[8] B.R. Holstein, D. Drechsel, B. Pasquini, and M. Vanderhaeghen, Phys. Rev. C61, 034316 (2000).

[9] See, e.g., T.R. Hemmert, B.R. Holstein, G. Knöchlein, and D. Drechsel, Phys. Rev. D62, 014013 (2000).

[10] J. Roche, Phys. Rev. Lett. 85, 708 (2000).

[11] T. R. Hemmert, B.R. Holstein, G. Knoechlein, and S. Scherer, Phys. Rev. Lett. 79, 22 (1997); Phys. Rev. D55, 2630 (1997)

[12] A. Metz and D. Drechsel, Z. Phys. A356, 351 (1996).

[13] M Vanderhaeghen, Phys. Lett. B368, 13 (1996)

[14] P. Guichon, G.Q. Liu, and A.W. Thomas, Aust. J. Phys. 49 (1996)

[15] Bates experiment 97-03, J. Shaw and R. Miskimen, spokesmen. 
[16] B. Pasquini, D. Drechsel, M. Gorchtein, A. Metz, and M. Vanderhaeghen, hep-ph/0007144.

[17] X. Ji and J. Osborne, hep-ph/9901410.

[18] S.B. Gerasimov, Sov. J. Nuc. Phys. 2, 430 (1966); S.D. Drell and A.C. Hearn, Phys. Rev. Lett. 16, 908 (1966).

[19] J.D. Bjorken, Phys. Rev. 148, 1467 (1966). 